\title{
Complex Visual Behavior with Simple Brains: Co-evolutionary Active Vision and Feature Selection
}

\author{
Dario Floreano (Dario.Floreano@epfl.ch) \\ Autonomous Systems Laboratory \\ Swiss Federal Institute of Technology Lausanne \\ http://asl.epfl.ch
}

\begin{abstract}
Brains are characterized by limited bandwidth and computational resources. At any point in time, we can focus our attention only to a limited set of features or objects. One of the most remarkable -and often neglecteddifferences between machine vision and biological vision is that computers are often asked to process an entire image in one shot and produce an immediate answer whereas animals are free to explore the image over time searching for features and dynamically integrating information over time.

I will show that the co-evolution of active vision and feature selection can greatly simplified computational complexity of visual performance. Each of these processes has been investigated and adopted in machine vision. Active vision is the sequential and interactive process of selecting and analyzing parts of a visual scene [Bajcsy, 1988, Ballard, 1991]. Feature selection instead is the development of sensitivity to relevant features in the visual scene to which the system selectively responds [Hancock et al., 1992, e.g.]. However, the combination of active vision and feature selection is still largely unexplored.
\end{abstract}

We carried out a series of experiments on co-evolution of active vision and feature selection for behavioral systems equipped with primitive retinal systems and deliberately simple neural architectures. In a first set of experiments, we show that sensitivity to very simple features is co-evolved with, and exploited by, active vision to perform complex shape discrimination. We also show that such discrimination problem is very difficult for a similar vision system without active behavior. In a second set of experiments, we apply the same co-evolutionary method and architecture for driving a simulated car over roads in the Swiss alps and show that active vision is exploited to locate and fixate simple features while driving the car. In a third set of experiments, we apply once again the same co-evolutionary method and architecture to an autonomous robot equipped with a pan/tilt camera that is asked to navigate in an arena located in an office environment. Evolved robots exploit active vision and simple features to direct their gaze at invariant features of the environment and perform collision-free navigation. In a fourth set of experiments, we apply this methodology to an all-terrain robot with a static, but large, field of view that must navigate in a rugged terrain. Here again, the system becomes sensitive to a set of simple visual fea-

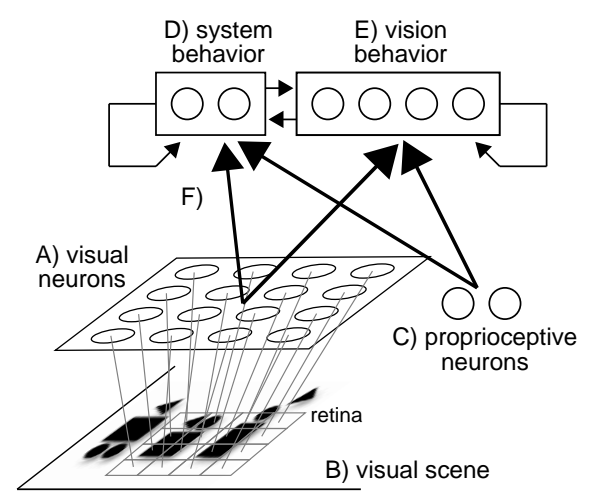

Figure 1: The architecture is composed of A) a grid of visual neurons with non-overlapping receptive fields whose activation is given by B) the grey level of the corresponding pixels in the image; C) a set of proprioceptive neurons that provide information about the movement of the vision system; D) a set of output neurons that determine the behavior of the system (pattern recognition, car driving, robot navigation); E) a set of output neurons that determine the behavior of the vision system; F) a set of evolvable synaptic connections. The number of neurons in each sub-system can vary according to the experimental settings.

tures that are maintained within the retine by the active vision mechanisms.

\section{References}

[Bajcsy, 1988] Bajcsy, R. (1988). Active Perception. Proceedings of the IEEE, 76:996-1005.

[Ballard, 1991] Ballard, D. H. (1991). Animate vision. Artificial Intelligence, 48:57-86.

[Hancock et al., 1992] Hancock, P. J., Baddeley, R. J., and S., S. L. (1992). The principal components of natural images. Network, 3:61-70. 